\title{
The interaction of denzimol (a new anticonvulsant) with carbamazepine and phenytoin
}

\author{
PN PATSALOS, SD SHORVON, AA ELYAS, G SMITH \\ From the The Chalfont Centre for Epilepsy, The University Department of Neurology, The Institute of \\ Neurology, and the Department of Chemical Pathology, The National Hospitals for Nervous Diseases, \\ London, UK
}

SUMMARY Denzimol, a new anticonvulsant drug, is currently undergoing clinical evaluation. In this paper we report its use in six patients who were also taking carbamazepine and two patients taking phenytoin. There was a striking elevation of serum carbamazepine, carbamazepine-10, 11 epoxide and phenytoin concentrations in all patients on the addition of denzimol therapy. The interaction with carbamazepine is greater in severity than any other reported to date and denzimol's interaction with both carbamazepine and phenytoin is likely to prove of major clinical significance.

Denzimol, an imidazole derivative $(\mathrm{N}-[\beta-[4(\beta-$ phenylethyl)phenyl]- $\beta$-hydroxyethyl]imidazole hydrochloride), is a new anticonvulsant drug,,$^{1-3}$ currently undergoing clinical trials in various countries. Its anticonvulsant profile assessed by a standard battery of laboratory tests, suggests that the drug may have a spectrum of action in clinical usage similar to that of carbamazepine and phenytoin.' In this paper we present the results of a hitherto unrecognised interaction of denzimol with both carbamazepine and phenytoin.

\section{Patients and methods}

Denzimol was assessed in an open clinical trial as adjunctive therapy in eight in-patients with severe intractable generalised or partial epilepsy, six of whom were taking carbamazepine and two phenytoin. Twice weekly blood sampling of fasting patients was performed between 8-9 am prior to morning dosage. Serum carbamazepine, phenytoin, phenobarbitone and valproic acid concentrations were measured using competitive binding immunoassay (EMIT; Syva UK). Confirmatory serum carbamazepine and carbamazepine-10, 11 epoxide measurements were made using high pressure liquid chromatography, according to the method of Elyas et al. ${ }^{4}$

Address for reprint requests: Dr S D Shorvon, The University Department of Neurology, Institute of Neurology, The National Hospitals, Queen Square, London WC1 3BG,UK

Received 17 April 1984 and in revised form 6 September 1984. Accepted 18 September 1984

\section{Results}

On initiation of denzimol therapy, in all six patients there was a marked rise in carbamazepine and

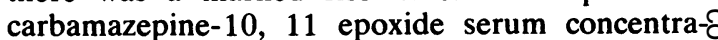
tions and a slight fall in the carbamazepine/epoxide ratio (figs 1-6). The rise in carbamazepine concen trations necessitated a reduction in carbamazepine dosage in four of the six patients, but in spite of this the mean baseline to peak rise in carbamazepine concentrations was $114 \%$ (range $50-142 \%$ ). Acute toxic side effects were experienced in all six patients;

Table 1 Toxic side effects in six patients taking carbamazepine

\begin{tabular}{ll}
\hline Patient: RW & Ataxia \\
& Diplopia \\
& Headache \\
& Dizziness \\
& Lethargy \\
& Dysarthria \\
Latient: MZ & Lethargy \\
Patient: SM & Ataxia \\
& Lethargy \\
& Nausea \\
& Vomiting \\
Patient: GB & Ataxia \\
& Diplopia \\
& Lethargy \\
& Nausea \\
Patient: AK & Lethargy \\
Patient: TN & Ataxia \\
& Nystagmus \\
& Dysarthria \\
& Dizziness \\
& Lethargy \\
\hline
\end{tabular}

(Time course of these side effects indicated on figures 1-6) 

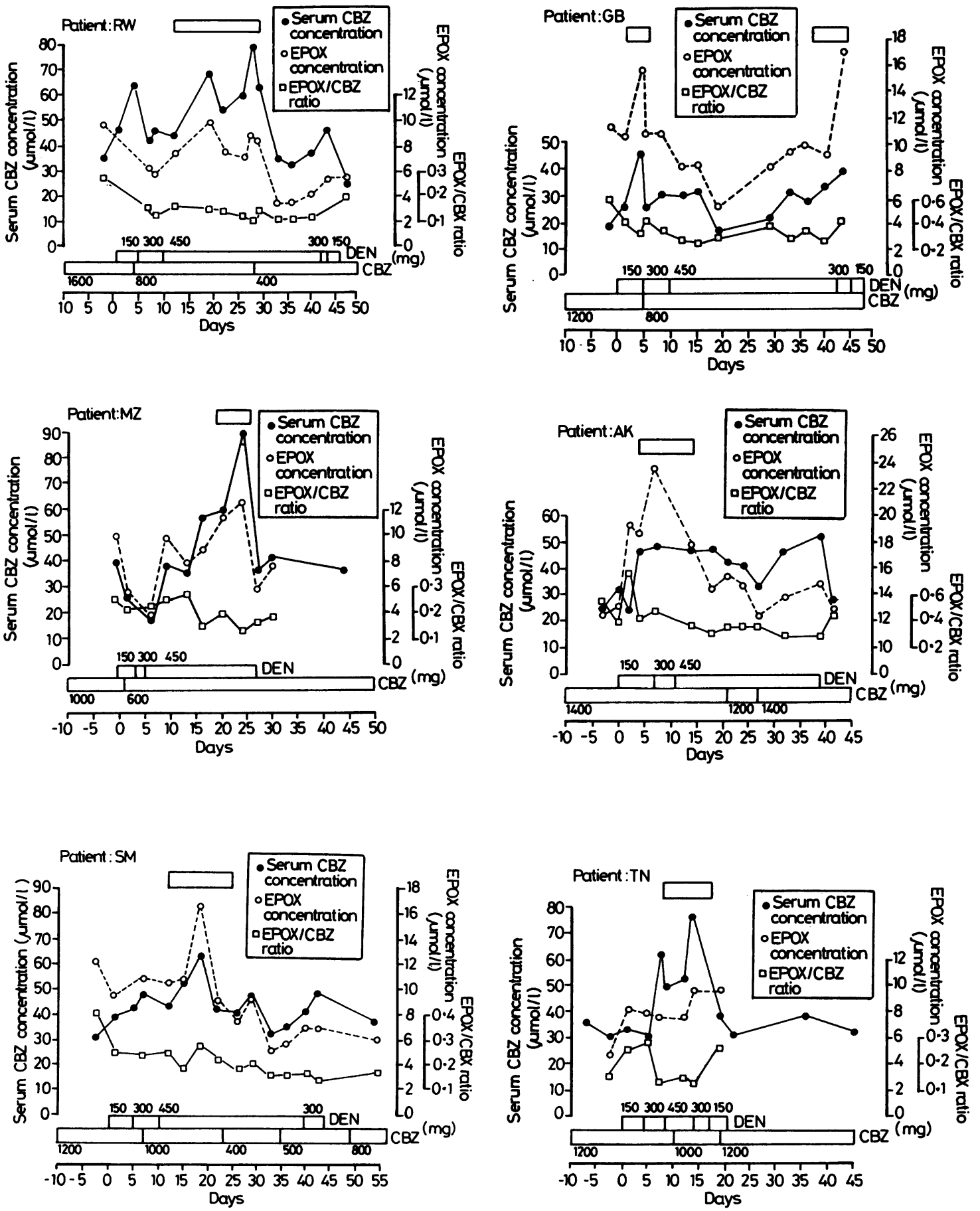

Figs 1-6 Graphical presentation, over time, of denzimol (DEN) and carbamazepine (CBZ) dosage and the serum concentrations of carbamazepine and carbamazepine-10, 11 epoxide (EPOX) and the carbamazepine-10, 11 epoxidel carbamazepine ratio (EPOX/CBZ) in 6 patients. The bars indicate the period of observed side effects (listed in table 1). 
Table 2 Effect of denzimol therapy on serum phenytoin concentration (two patients)

\begin{tabular}{|c|c|c|c|}
\hline & $\begin{array}{l}\text { Denzimol Dose } \\
\text { (mg/day) }\end{array}$ & $\begin{array}{l}\text { Phenytoin se } \\
\text { ( } \mu \text { molll) } \\
\text { Patient-JG }\end{array}$ & Patient-IJ \\
\hline \multirow{3}{*}{$\begin{array}{l}\text { Pretrial } \\
\text { Week } 1\end{array}$} & 0 & 23 & 32 \\
\hline & 150 & 23 & 40 \\
\hline & 300 & 32 & 38 \\
\hline \multirow{2}{*}{ Week 2} & 450 & 31 & 51 \\
\hline & 450 & 35 & 42 \\
\hline \multirow{2}{*}{ Week 3} & 450 & 37 & 57 \\
\hline & 450 & 46 & 63 \\
\hline \multirow[t]{2}{*}{ Week 4} & 450 & 53 & 62 \\
\hline & 450 & 49 & 65 \\
\hline \multirow[t]{2}{*}{ Week 5} & 450 & 56 & 67 \\
\hline & 450 & 63 & 63 \\
\hline
\end{tabular}

(Phenytoin dosage: Patient JG-200 mg/day, Patient IJ-400 mg/day)

these are listed in table 1 and their time relationships shown in figs $1-6$.

The dosage of denzimol and phenytoin and the phenytoin serum concentrations in the two patients taking phenytoin are shown in table 2 . In both cases serum phenytoin concentrations rose gradually over the period of study and by 5 weeks of denzimol therapy the percentage rise from the baseline values was $97 \%$ and $174 \%$. Seven patients were comedicated sodium valproate and three with primidone, but there was no significant change in serum valproic acid or phenobarbitone concentrations, when denzimol was added.

\section{Discussion}

Drug interactions involving anticonvulsants are frequent but usually minor and of little practical relevance. In this paper we report a severe interaction between denzimol and carbamazepine and this is likely to be an important consideration in its clinical usage. In all six patients taking carbamazepine a rapid and marked rise in carbamazepine serum concentrations was demonstrated. As drug dosages had to be reduced in four patients, the full extent and timing of this interaction cannot be determined but an indication of its severity is that in the five patients who continued on denzimol and in whom carbamazepine concentrations eventually returned to pre-denzimol levels, the mean reduction in carbamazepine dosage was $47 \%$ (range 14-75\%). The exact mechanism of this effect could not be deduced from this study but the rapidity and extent of the rise may suggest an interference with carbamazepine metabolism. The 10, 11 epoxidation of carbamazepine is the major first step in the metabolism of carbamazepine. ${ }^{5}$ On denzimol treatment the epoxide/carbamazepine ratio was slightly reduced, suggesting inhibition at this level, but as serum concentrations of both carbamazepine and carbamazepine-10, 11 epoxide rose and fell simultaneously a simple block at this metabolic step does not fully explain these observations.

Phenytoin serum concentrations also rose in both patients co-medicated with denzimol. The rate of rise was slower than that of carbamazepine and at the end of five weeks of denzimol therapy levels had risen by $97 \%$ and $174 \%$ and were continuing to rise. As phenytoin and carbamazepine undergo metabolic degradation by similar hepatic microsomal enzymes this further suggests a metabolic interaction. The slower rise is serum phenytoin concentrations probably reflects its slower metabolic handling.

Inhibition of carbamazepine metabolism by other drugs has been reported (for example propox yphene $^{6}$ and triacetyloleandomycin ${ }^{7}$ ) but the mag $\Omega$ nitude of these interactions is smaller than that reported here. The extent of denzimol's interactiog with phenytoin is similar to that seen with phenytoin? and sulthiame. ${ }^{x y}$

In the six patients taking carbamazepine, toxic side effects were noted at times when the carbamazepine levels were very high and these resolved when the carbamazepine dosage was lowered. These side effects were likely to be due to intoxication by carbamazepine (or its epoxide) and not to denzimol per se. No interaction between denzimol and sodium valproate was noted in any of the seven patients taking this drug and it is noteworthy that valproate (a short chain branched fatty acid) undergoes metabolic degradation which is very different from that of other commonly used anticonvulsant drugs. ${ }^{10}$

Other anticonvulsants with similar chemical structures to denzimol are in the early stage of clinical development and similar interactions should be anticipated. Whether these findings will limit the value of such drugs in routine clinical practice remains to be seen.

We are grateful for the assistance of Recordati Limited, the manufactures of denzimol and to Mrs Berenice Jones for the preparation of the manuscript. 


\section{References}

' Graziani G, Tirone F, Barbadoro E, Testa R. Denzimol, a new anticonvulsant drug. I. General anticonvulsant profile. Drug Res 1983;33:1155-60.

${ }^{2}$ Graziani G, Cazzulani P, Luca C, Nava G, Testa R. Denzimol, a new anticonvulsant drug. II. General pharmacological activities. Drug Res 1983;33:11617.

${ }^{3}$ Graziani G, Barbadoro E, Testa R. Denzimol, a new anticonvulsant drug. III. Toxicological evaluation. Drug Res 1983;33:1168-73.

+ Elyas AA, Ratnaraj N, Goldberg VD, Lascelles PT. Routine monitoring of carbamazepine and carbamazepine-10, 11 epoxide in plasma by high performance liquid chromatography using 10methoxycarbamazepine as internal standard. $J$ Chromatgr 1982;231:93-101. s Sillanpaa M. Carbamazepine. Pharmacology and clinical uses. Acta Neurol Scand 1981;64 (suppl 88):1-202.

- Dam M, Kristensen CB, Hansen BS, Christiansen J. Interaction between carbamazepine and propoxyphene in man. Acta Neurol Scand 1977;56:603-7.

${ }^{7}$ Mesdjian E, Dravet C, Genroud B, Roger J. Carbamazepine intoxication due to triacetyloleandomycin administration in epileptic patients. Epilepsia 1980;21:489-96.

${ }^{8}$ Hansen JM, Kristensen M, Skorsted L. Sulthiame (Ospolot) an inhibitor of diphenylhydantoin metabolism. Epilepsia 1968;9:17-22.

' Houghton GW, Richens A. Inhibition of phenytoin metabolism by sulthiame in epileptic patients. $\mathrm{Br} J$ Clin Pharmacol 1974;1:59-66.

${ }^{10}$ Ferrandes B, Eymard P. Metabolism of valproate sodium in rabbit, rat, dog and man. Epilepsia 1977; 18: 169-82. 